\title{
Nature and standards of gastrointestinal and liver services in the United Kingdom
}

M J G Farthing, R Williams, C H J Swan, A Burroughs, R C Heading, J A Dodge, R I Russell, C W Venables, R Dick, R Burnham, R Leicester, G Neale, E T Swarbrick, P D Fairclough, R Jones, N P Melia

\section{Purpose of the working party}

1.1 To describe the scope of major digestive and liver disorders and identify changes in patterns of disease.

1.2 To identify diagnostic and therapeutic services required to manage these disorders in the United Kingdom.

1.3 To describe the facilities and staffing required to provide these services.

1.4 To examine the training requirements for medical, nursing, and other support staff.

1.5 To define the part that audit and research should play in the provision and maintenance of high quality gastrointestinal and liver services.

(Gut 1993; 34: 1728-1739)

\section{Introduction}

Since the publication of the document Provision of a Gastroenterological Service in a District General Hospital, a monograph prepared by a working party of the Gastroenterological Liaison Committee with the Department of Health and Social Security in 1979,,$^{1}$ diagnostic and therapeutic gastroenterological and liver services have developed at a great pace. New and more effective methods have been introduced in the diagnosis and treatment of common disorders of the gastrointestinal tract. Endoscopy in particular has become an increasingly important part of the gastroenterologist's workload. Endoscopic techniques are now used in the diagnosis and control of upper gastrointestinal bleeding, in the non-surgical treatment of benign and malignant strictures of the oesophagus, in the removal of gall stones from the biliary tract, in the management of extrahepatic cholestatic jaundice, and in the diagnosis of colonic disorders including inflammatory bowel disease, colonic bleeding, and in screening for colorectal cancer. As a consequence, requests for diagnostic gastrointestinal radiological examinations have decreased and some major gastrointestinal and biliary tract surgical procedures can be avoided. Expertise in interventional radiology is, however, also required to support these therapeutic advances. Hepatological areas in which specific clinical facilities and expertise is required are the administration of anti-viral treatment for chronic viral hepatitis, management of portal hypertension, intensive therapy for fulminant liver failure and liver transplantation - a major development in the care of patients with other- wise untreatable, end stage liver disease. The discovery of Helicobacter pylori has increased the complexity of the treatment options for peptic ulcer disease and gastritis, the management of which may require special expertise.

Gastroenterologists (both medical and surgical) have taken an increasingly active role in the provision of hospital and community based nutrition services including the organisation of hospital nutrition teams, planning enteral and intravenous nutrition strategies for individual patients, placement of central venous cannulas, insertion of feeding gastrostomies, and monitoring treatment. As a result the total workload of the gastroenterologist has escalated substantially during the past decade, which has only partly been met by an increase in the number of consultant physicians with an interest in gastroenterology, which has risen from 245 in 1980 to 340 at the end of 1991 (Appendix I). Although on average there is now one gastroenterologist for 1:150 000 population there are regional differences throughout the UK. Seventeen health districts still do not have a gastroenterologist, 37 have only one gastroenterologist serving populations of more than 250000 , and 34 health districts have one gastroenterologist serving populations between 200-250 000 (Appendix II). These figures compare unfavourably with western Europe $(1: 60000)$ and the USA $(1: 33000)$. The disparity is further heightened by the fact that most gastroenterologists in western Europe and USA no longer make a major contribution to the provision of acute general medical services. Eighty six per cent of gastroenterologists in the UK continue to take a share of the acute general medical admissions (Royal College of Physicians survey).

The purpose of this report is to detail the requirements of gastrointestinal and liver services at a district and a regional level, to define standards of care in gastroenterology and related specialities, to anticipate medical, nursing and ancillary manpower requirements for the future, and the needs for training and research in gastroenterology.

\section{Digestive and liver diseases in the United}

\section{Kingdom}

Disorders of the digestive tract and liver continue to be an important cause of morbidity and mortality in the population, and in consequence represent a major caseload for general 
practitioners and hospital doctors. In broad terms, $10 \%$ of patients seen in general practice have digestive complaints and about $10 \%$ of hospital admission are a result of diseases of the gastrointestinal tract. The common disorders, peptic ulcer and related disorders affect 1 in 15 of the population at any one time. Colonic, gastric, oesophageal and pancreatic cancer continue to be important causes of death in the United Kingdom (Appendix III). Gastric cancer accounts for 8-10 000 deaths/annum, which is about $10 \%$ of all deaths from cancer in the UK. Colorectal cancer kills 17000 each year, being responsible for the deaths of seven times more women than those dying as a result of cervical cancer. Gastrointestinal cancer accounts for about $30 \%$ of all cancer deaths in England and Wales. Inflammatory bowel diseases, ulcerative colitis, and Crohn's disease affect at least 100000 people in the UK and the latter is increasing in incidence. Upper gastrointestinal haemorrhage is a major cause of hospital admission with a mortality of 5-15\%. Functional bowel disorders such as the irritable bowel syndrome probably occur in $20 \%$ or more of the UK population and constitutes $30-40 \%$ of the outpatient gastroenterology workload.

It is estimated that there are at least 750000 alcohol abusers in the UK, a substantial proportion of whom will go on to develop chronic liver disease, acute and chronic pancreatitis, and pancreatic insufficiency. About $50 \%$ of liver biopsies in alcohol abusers with abnormal liver biochemical tests show established cirrhosis and $25 \%$ alcoholic hepatitis. Death rates for cirrhosis in England and Wales have increased by more than $50 \%$ in the last 30 years and death rates attributable to alcohol have increased eightfold. Gastroenterologists often participate in the care of other patients with alcohol related problems. The demands on the service can be considerable as alcohol is implicated in up to $20 \%$ of acute surgical and orthopaedic hospital admissions. The prevalence of other liver disorders such as primary biliary cirrhosis, primary sclerosing cholangitis, and haemochromatosis have previously been underestimated, and the first is now the most common indication for liver transplantation in the UK. A proportion of alcoholic patients with cirrhosis are suitable candidates for liver transplantation and this will increase the need for these services. Gall stone disease continues to be an important problem in the United Kingdom with more than $20 \%$ of women and $11.5 \%$ of men over the age of 60 years having had gall stones or have had cholecystectomy. The incidence of many conditions such as chronic inflammatory bowel disease, gastro-oesophageal reflux disease especially in the elderly, alcohol related gastrointestinal disorders, cancer of the oesophagus, colon and pancreas, and adverse drug effects on the gastrointestinal tract and liver are almost certainly on the increase. For example, $20-30 \%$ of acute gastrointestinal haemorrhages are related to the ingestion of non-steroidal antiinflammatory drugs. be managed on an outpatient basis and this constitutes a substantial proportion of the gastroenterologist's workload. Most of the gastroenterologist's work is in the outpatient clinic and in day case endoscopy. The medical gastroenterologist also continues to fulfil an active role in the provision of acute general medical services including both inpatients and outpatients. District general hospital physicians admit/ discharge 680-700 acute admissions each year. ${ }^{2}$ Two regional surveys of district hospital physicians with a special interest in gastroenterology, however, reported 900 and 750-1130 admissions annually ${ }^{2}$ and a recent survey of 17 regions in the UK carried out by the British Society of Gastroenterology confirmed these earlier findings when 135 gastroenterologists had 133920 deaths or discharges, averaging 992 inpatient episodes/year, while the average for other physicians was $679 .^{3}$ When day case admissions are included annual discharges for gastroenterologists increased to 1553 compared with 871 for other physicians. These additional 682 cases represent the workload of gastrointestinal endoscopy. Thus, while the physician/ gastroenterologist continues to contribute equally to acute general medical services, workload has risen substantially because of the routine endoscopic commitments. In addition, out of hours endoscopy has also increased because of the endoscopist's increasing role in the diagnosis and treatment of gastrointestinal bleeding and jaundice.

\section{Gastrointestinal and liver services}

\subsection{INPATIENT}

Gastrointestinal disorders account for $20-30 \%$ of the total number of inpatients cared for by gastroenterologists. ${ }^{2}$ As outpatient management is now highly developed in gastroenterology, gastrointestinal inpatients often require disproportionally more medical and nursing time than other patients because of the severity of their illness. Typical reasons for inpatient care include serious complications of inflammatory bowel disease including nutritional insufficiency, acute gastrointestinal bleeding, severe pancreatitis, obstructive jaundice, complicated gastrointestinal surgical problems, patients with fulminant hepatic failure, those having liver transplantation, and patients with major complications of chronic liver disease such as ascites and variceal bleeding. Many of these patients require integrated management strategies including physicians, surgeons, radiotherapists, and interventional radiologists. Medical and nursing care is delivered most efficiently in a multidisciplinary inpatient facility. ${ }^{4}$ Access to intensive therapy unit beds is required for patients with hepatic failure and liver transplantation and complex gastrointestinal surgery; high dependency beds are required for some patients with the complications of liver disease and severe pancreatic disease. 
week in the outpatient department seeing 1000 1500 new patients each year. ${ }^{23}$ Most of these patients $(70-100 \%)$ will have gastrointestinal or hepatic disorders. About $30-40 \%$ of new outpatients will have functional bowel disorders including the irritable bowel syndrome and nonulcer dyspepsia. Consultations with these patients tend to be longer than those with organic disease as environmental stresses and psychological factors need to be explored. Patients with chronic inflammatory bowel disorders and those with chronic liver disease because of the specialised nature of these disorders, tend to use the hospital outpatient department rather than their general practitioner as the main route for seeking medical advice about their condition. Physicians and surgeons in gastroenterology and hepatology commonly run joint clinics to facilitate the integrated management of conditions such as chronic inflammatory bowel disease, biliary disease, upper gastrointestinal cancer, and liver transplantation. The multidisciplinary approach to outpatient care will probably increase in those disorders in which combined management is the rule.

\subsection{THE INTERFACE WITH GENERAL PRACTICE} Initial investigation of patients with digestive disorders commonly takes place in general practice and although small numbers of general practitioners are developing flexible sigmoidoscopy and even gastroscopy for their patients there is increasing pressure for open access to hospital investigative services, particularly upper gastrointestinal endoscopy and flexible sigmoidoscopy and colonoscopy, and 'fast track' access for patients presenting in general practice with symptoms requiring urgent assessment. Almost 200 general practitioners are employed as hospital practitioners or clinical assistants in endoscopy, making a contribution in most district general hospitals. The development of medical audit and of standards for the care of common conditions will increase the requirement for the establishment of clinical guidelines and protocols for the care of digestive disorders agreed between practitioners and specialists.

\subsection{DAY CARE}

About $40 \%$ of the workload of a physician/ gastroenterologist will be day cases including almost all diagnostic and most therapeutic procedures. ${ }^{3}$ This comparatively high figure is the result of changing practicés in endoscopy where patients are no longer admitted overnight for preparation for colonoscopy or endoscopic retrograde cholangiopancreatography (ERCP). With the continued development of endoscopic techniques it is anticipated that this proportion of the workload will continue to increase. Unquestionably, the day care approach in gastroenterology is economically sound and produces minimal disruption to the life of the patient. It places increased demands, however, on administrative services to operate such a system. Adequate reception, preparation, and recovery facilities are essential to ensure that this expanding area of health care remains safe and acceptable to the patient. Most other gastrointestinal investigations such as liver, pancreatic and intestinal biopsies, gastrointestinal manometry, oesophageal $\mathrm{pH}$ monitoring, anorectal physiological studies, non-invasive testing for bacterial overgrowth, pancreatic function tests, etc can be performed on a day case basis. Venesection for iron overload, iron infusion for iron deficiency, etc are usually performed as a day case, unless complications arise.

\subsection{DIAGNOSTIC SERVICES}

Although the gastroenterologist is inevitably dependent on good radiological and laboratory services, many gastroenterology units have established their own comprehensive array of diagnostic tests and procedures, which makes them relatively autonomous. Endoscopy has been the mainstay of diagnosis in patients with gastrointestinal disorders but most departments have set up in house or have easy access to a range of non-endoscopic investigations, which are vital for the management of patients with gastrointestinal disorders (5.5.2). Most such tests have been set up by individual consultants and their junior staff on an ad hoc basis, often serviced by research fellows and research nurses but not funded by the NHS. The situation is currently unsatisfactory but should improve with an increase in the numbers of clinical measurement technicians in gastroenterology, and with the establishment of appropriate budgets to fund non-endoscopic diagnostic services in gastroenterology.

\subsubsection{Endoscopy}

A detailed account of Provision of gastrointestinal endoscopy and related services for a district general hospital was published in $1990^{5}$ and prepared by a working party set up by the clinical services committee of the British Society of Gastroenterology. This report sets out the application of endoscopy in clinical gastroenterology, defines standards for staff, patient care, and equipment, and makes detailed recommendations on the planning, staffing, and equipment necessary for a purpose built endoscopy unit, which should be available in every district general hospital. The development of flexible endoscopes has now made it possible to examine the entire gastrointestinal tract, including the biliary and pancreatic ducts (Appendix IV). Endoscopic examination of the upper gastrointestinal tract and the colon has tended to replace barium meal and barium enema examinations. In addition to visualising the oesophagus, stomach, and intestine directly, it is possible to take biopsy specimens for histological and microbiological examination to increase diagnostic precision. Therapeutic endoscopy continues to develop. Dilatation of strictures, removal of colonic polyps, and extraction of gall stones from the bile ducts have now replaced the equivalent surgical procedures. These procedures are minimally invasive and highly cost effective. First line treatment for variceal and non-variceal bleeding from the upper gastrointestinal tract is now endoscopy based. 
The number of endoscopic examinations performed each year continues to rise. Epidemiological data and audit of the work of established endoscopy units shows that the annual requirements/head of population for upper gastrointestinal endoscopy is $1: 100$, for flexible sigmoidoscopy and colonoscopy each is 1:500, and for ERCP 1:1000. For a district general hospital serving a population of 250000 this is approximately 3500 procedures each year. An endoscopy service should be consultant led. At least 10 clinical sessions including most of the simple upper gastrointestinal diagnostic procedures each week are required, six or more of which should be consultant sessions. The remaining 2-4 sessions, including most of the simple gastrointestinal diagnostic procedures can be covered by clinical assistants or staff specialists.

There is an increasing requirement for general practitioners to have open access to endoscopic procedures (gastroscopy, rigid, and flexible sigmoidoscopy) and a desirability for 'same day' endoscopy when patients are seen by hospital gastroenterologists in the outpatient department. An endoscopy service requires a purpose built suite of rooms, trained specialised nurses possibly including an endoscopy department assistant (analogous to an operating department assistant), and appropriate secretarial and clerical support for appointments, record keeping, reports and audit, all of which should be computer based (sections 8.3, 9.3, 9.4, and 9.6). An endoscopy unit must be supported by an adequate day care facility, appropriate portering services, and thus achieve the same status as a surgical operating suite.

Safety in endoscopy has received considerable attention recently such that it is now vital that units should be provided with adequate monitoring equipment, supplementary oxygen, and resuscitation equipment and adequately ventilated, cleaning, and disinfection facilities for endoscopes.

Appendix V summarises the availability in the UK of some of the more commonly used diagnostic and therapeutic endoscopic procedures. A detailed account of the results of this survey will be published as a separate report.

\subsubsection{Non-endoscopic gastrointestinal investigations}

In addition to endoscopy, the gastroenterologist requires access to a variety of other investigations that have become an established part of their clinical practice (Appendix VI). District general hospitals will not necessarily have all of these tests established in house, but they should be easily available on a sub-regional basis established through a network of district general hospitals or the local teaching hospital. With the advent of gastrointestinal endoscopy and biopsy, the time honoured functional tests of absorption (faecal fat excretion, xylose absorption test, Schilling test, etc) have become less widely used for screening purposes, although still have their specific indications. Functional assessment of oesophageal motility and 24 hour ambulatory $\mathrm{pH}$ monitoring contribute to the diagnosis of oesophageal disease and the choice of treatment.
Anorectal manometry and electophysiological evaluation are increasingly used for the assessment of constipated and incontinent patients, although generally only available in specialised units. Glucose and lactulose hydrogen breath tests are widely available for assessment of bacterial overgrowth and small intestinal transit time respectively, and most units require access to a test of pancreatic function (pancreolauryl, Lundh test, or PABA test). There has been an increasing requirement to have access to noninvasive approaches to the diagnosis of $H$ pylori infection. ${ }^{14} \mathrm{C}$ or ${ }^{13} \mathrm{C}$ urea breath tests have been established in some departments while others have used $H$ pylori serology. As more effective eradication procedures are developed, noninvasive tests for $H$ pylori will assume increasing importance. Access to specific antibody tests for screening for coeliac disease (anti-gliadin antireticulin and anti-endomysial antibodies) is also desirable.

Appendix VII summarises the availability in the UK of some of the more commonly used nonendoscopic tests in gastrointestinal disorders. A detailed account of the results of this survey will be published as a separate report.

Those involved in hepatology will require facilities for full virological study of patients with hepatitis (hepatitis viruses $A, B, C$, etc) including HBV-DNA and HCV-RNA, tumour markers, autoantibody profiles, liver, iron and copper quantifications, and in those centres carrying out liver transplantation, the assays for measuring plasma concentrations of immunosuppressive drugs such as cyclosporin $A$. In addition radiological services specific to liver disease are essential for the investigation and treatment of patients. These are specialist services, so that transfer of patients will become necessary within a reasonable distance. The investigations include splanchnic arteriography (selective mesenteric, coeliac, and hepatic arteriography) for diagnosis and chemoembolisation of hepatic tumours and presurgical assessment of the splanchnic circulation. Hepatic venography and hepatic venous pressure measurements are required for diagnosis and monitoring of portal hypertension. A further radiological procedure is the transjugular intrahepatic portal systemic stent shunt, which already has a therapeutic role in some patients with bleeding varices.

Gastroenterologists are highly dependent on good quality gastrointestinal radiological services (including interventional techniques), histopathology, and microbiology and it is desirable that close links are developed with these respective departments to ensure the development of effective services without duplication of effort. Shared nurses with ability in both endoscopic and percutaneous procedures are required and there should be full nursing cover for each department at all times. Many gastroenterology units have regular interdepartmental meetings with radiologists and histopathologists to review the results of diagnostic procedures and to facilitate continuing education and audit. The full integration of these services is essential for the provision of high quality care in gastroenterology and hepatology. In addition there is a need for the active support of specially 
trained nursing staff and intensive care support.

\subsection{THERAPEUTIC SERVICES}

\subsubsection{Endoscopy}

Appendix $\mathrm{V}$ details the range of therapeutic endoscopic procedures. Many of these procedures are performed on a day case basis within the endoscopy unit while others such as therapeutic ERCP, dilatation of malignant oesophageal strictures, and placement of oesophageal endoprostheses require $x$ ray screening and direct collaboration with the department of radiology. Rapidly developing areas of therapeutic endoscopy, in addition to gall stone removal and stenting of strictures in the biliary tract, include tumour bulk reducing procedures with laser, diathermy or ethanol injection, photodynamic therapy, and brachytherapy (endoradiotherapy). A few specialised centres have lithotripsy for gall bladder stones but this is probably not widely applicable and thus will be restricted to a few specialised regional centres.

\subsubsection{Clinical nutrition}

It is now increasingly recognised that chronic diseases, particularly those of the gastrointestina tract, are almost inextricably linked with some degree of nutritional insufficiency. Publication of $A$ positive approach to nutrition as treatment by the King's Fund Centre ${ }^{6}$ has heightened awareness of the importance of the added risk that undernutrition brings to patients, the ways in which undernutrition can be detected, and the importance of providing appropriate nutritional support both in the hospital and at home. Many gastroenterologists (physicians and surgeons) directly participate in nutritional assessment and nutritional support and many lead hospital clinical nutrition teams and coordinate a multidisciplinary team consisting of clinicians, nurses, dietitians, and pharmacists. Close liaison needs to be established with departments of chemical pathology, microbiology, and dietetics. In addition, the clinician's specific responsibilities include nutritional assessment and identification of the most appropriate approach to nutritional support, placement of gastrostomy and intravenous catheters, prescription of parenteral nutrition solutions, planning enteral and other nutritional regimens, overall responsibility for the nutritional aspects of care of referred patients, and for monitoring the results of treatment. The average workload of a nutrition team in a 740 bed district general hospital has recently been assessed at 3000 patient days for enteral tube feeding, 240 patient days for intravenous nutritional support, and 1200 patient days for home enteral tube feeding. ${ }^{7}$ Recent evidence suggests that there are also important economic advantages in creating a team based nutrition service.

Approximately 150 patients in the United Kingdom currently receive intravenous nutrition at home. This is a highly specialised service and a comparatively small number of centres in the United Kingdom provide the medical and nursing support for these patients. Home in- travenous nutrition will continue to be supervised by a limited number of centres led by a gastrointestinal physician or surgeon with a nurse specialist in nutrition. Home enteral nutrition, however, is increasing and appropriate organisational structures are being established between hospital and the community. All patients on artificial nutrition support require to be closely monitored by gastroenterologists, often in dedicated nutrition clinics in liaison with chemical pathologists, dietitians, and nutrition nurses. There is a need to streamline the funding procedures for enteral feeding preparations and equipment in cooperation with the community services. Some progress is already being made in this matter. Endoscopic guided percutaneous gastrostomy is a growing technique for providing enteral nutrition and requires expertise of both the endoscopist and radiologist.

\subsubsection{Stoma care}

Nurse specialists in stoma care are an essential component of gastrointestinal services in the hospital and community. Ideally stoma care nurses should work in close liaison with medical and surgical gastroenterology clinics. Stoma care nurses should be made aware of the decision making processes associated with surgery that will probably result in a stoma, to permit the patient to be appropriately prepared.

\subsubsection{Psychological services in gastroenterology}

A substantial proportion of outpatient gastroenterology involves functional disorders of the gastrointestinal tract some of which have an underlying psychological component. Many gastroenterologists have developed close links with a psychiatrist or clinical psychologist to assist in the assessment and treatment of such patients. Such services will probably be limited to centres where there is particular interest and experience in these disorders.

\section{Paediatric gastroenterology}

Paediatric gastroenterology has developed rapidly during the past 15 years although there are still NHS regions without recognised paediatric gastroenterologists. Among paediatric gastroenterologists there is concern that many children are still deprived of the opportunity of investigation by appropriate modern methods and that they may receive treatment that is suboptimal. With the development of the advanced endoscopic techniques that are now available for the investigation of gastrointestinal diseases in the paediatric age group and of new approaches to nutritional rehabilitation, it is vital that specialists in this area should be available to children with these disorders. The British Society of Paediatric Gastroenterology and Nutrition has recently examined regional services for paediatric gastroenterology and nutrition and produced a report. ${ }^{8}$ Because children are fundamentally different from adults, they should not be treated like 'mini-adults'. The report recommends that each region should have at least one identified referral centre for children with gastrointestinal, liver, and nutritional disorders. It recommends 
that children with these disorders should be managed in a dedicated children's unit with close collaboration with other specialists such as paediatric surgeons, radiologists, and child psychiatrists. Each referral centre should have a dedicated paediatric endoscopy unit but failing this there should be designated paediatric sessions in the general endoscopy unit. In addition, each regional centre should have the full range of other gastrointestinal investigations including oesophageal $\mathrm{pH}$ monitoring, hydrogen breath testing, tests of pancreatic function, and other specialist biochemical tests including mucosal disaccharidase activities, and an experienced nutrition support team.

\subsection{HEPATOLOGY}

Currently there are two major centres in the UK resourced supraregionally. Regional paediatric expertise in hepatology will be important to ensure early and appropriate referral to supraregional centres.

\subsection{PAEDIATRIC GASTROINTESTINAL SURGERY} Closely integrated medical and surgical management is essential for conditions such as severe gastro-oesophageal reflux, biliary atresia, inflammatory bowel disease, meconium ileus, and protracted diarrhoea after neonatal surgery. Endoscopy services and nutritional support again should be the joint responsibility of paediatric gastroenterologists and paediatric surgeons. All surgery in children under the age of two years should be performed in a regional paediatric surgical centre and never in adult units at any age. Appendix VIII lists conditions that could be referred to regional paediatric gastroenterology centres.

\section{Surgical gastroenterology}

The extent of a gastrointestinal surgery service at a district general hospital will depend upon the size of the hospital, the population it serves, and the number of general surgeons on its staff. All district general hospitals must provide a full gastrointestinal emergency service but the level of the service provided will vary. No unit should be expected to provide a complete service for every gastrointestinal problem. Surgical expertise in the management of rare but important gastrointestinal problems can only be developed by concentrating such cases in designated centres on a subregional, regional or national basis. It is considered that a surgical gastrointestinal service can be provided on a three level basis depending on the amount of specialised expertise that is required for any individual case.

\section{LEVEL 1}

This level must be available at all district general hospitals and provide a 24 hour emergency cover, irrespective of the size of the population it serves. It will require a minimum of two gastrointestinal trained surgeons to be available with two or three other surgeons to provide support for the emergency work. The latter may have major interests in other fields but must not be so specialised that they cannot support the two gastrointestinal surgeons with gastrointestinal emergencies. Similarly, the gastrointestinal surgeons will need to have a sufficiently wide training to support the other speciality interests when an emergency situation arises. Such a unit might be expected to have at least one and ideally two general physician/gastroenterologists on the staff.

Emergency surgery - these surgeons must be able to deal with acute appendicitis, intestinal obstructions, perforations of the bowel and other intra-abdominal organs, acute cholecystitis, hernias and their complications, diverticulitis, major upper gastrointestinal bleeding, resuscitation of acute pancreatitis, mesenteric infarction, volvulus, etc. To provide these services they would require access to upper gastrointestinal endoscopy and colonoscopy and be fully trained in emergency endoscopy.

Elective surgery - the type of 'elective surgery' performed in such a unit should be limited to those conditions/procedures that are seen commonly enough to maintain their expertise. They should not be expected to undertake complicated, difficult surgical procedures that require considerable experience, substantial anaesthetic/laboratory support, and expert nursing and junior medical staff support. Such problems are identified under level 2 .

\section{LEVEL 2}

This would be provided in a larger institution or group of hospitals with at least eight general surgeons on the staff, four of whom would have been trained in gastrointestinal surgery. This institution would be expected to provide some surgical services on a regional or subregional basis, receiving referrals from other small (level 1) district general hospitals surrounding their own district. The four gastrointestinal surgeons would work closely with at least two medical gastroenterologists and be supported by a full endoscopic service including diagnostic and therapeutic ERCP, intensive care including renal failure management, interventional radiology, and advanced biochemical and laboratory services. They would provide a range of specialised services dependent upon their experience and expertise. All of the gastrointestinal surgeons would take part in the emergency service in association with the other general surgeons and provide backup support for the other surgeons if a major gastrointestinal emergency was admitted. All would have sessional commitments to endoscopy but this could be focused to their area of interest such as colonoscopy for the coloproctologists and upper gastrointestinal endoscopy and ERCP for those surgeons interested in upper gastrointestinal and hepatobiliary/pancreatic surgery. The specialised services provided in such a unit would be expected to include oesophageal, oesophagogastric, revisional gastric, pancreatic, hepatic and biliary reconstructive, specialised colorectal and anal surgery. 
Elective emergency - the specialised surgical services covered by such a unit (depending on local expertise) might include oesophageal and oesophagogastric surgery; complicated biliary surgery (for example, strictures, tumours, difficult bile duct stones); hepatic and oesophageal variceal surgery; surgical management of acute and chronic pancreatitis; major pancreatic surgery for cancer and tumours; recurrent intestinal fistulas; recurrent unexplained gastrointestinal bleeding; severe complicated inflammatory bowel disease; trans anal surgery, and surgery for incontinence plus major laparoscopic surgery.

LEVEL 3

This type of unit would provide a highly specialised gastrointestinal surgical service on a regional or national basis. It would not necessarily provide a full range of gastrointestinal surgical services but would concentrate upon narrow areas, for example oesophageal varices management, liver resections; hepatic, pancreatic or intestinal transplantation; home intravenous nutrition; short bowel syndrome management, etc.

The gastrointestinal surgical service is logically stratified into levels depending on the patients' problems. It is vital that all concerned should be aware that not all district general hospitals can or should be expected to provide a full range of gastrointestinal surgical procedures. Referral to other surgical centres will always be likely, particularly when highly specialised, difficult surgical procedures are required to treat the condition.

\section{Facilities}

Gastrointestinal medicine and surgery should ideally be closely integrated in the care of inpatients and outpatients, both of which should be geographically allied to a purpose built endoscopy unit, clinical investigation unit for nonendoscopic gastrointestinal investigations, and the outpatient department. Proximity of the outpatient department to endoscopy will facilitate 'same day' investigations and a joint medical/ surgical gastrointestinal inpatient facility will optimise the shared care arrangements that have become desirable and unavoidable in many disorders of the gastrointestinal tract. Office accommodation for medical staff and secretaries should be provided adjacent to investigation and inpatient facilities.

\subsection{INPATIENT FACILITY}

The size of the inpatient unit would depend on the population served, the referral practice, and specific expertise of the gastrointestinal physicians and surgeons. Centres dealing with complicated inflammatory bowel disease, short bowel syndrome, complex nutritional problems, organ transplantation, and complicated pancreatic disease will require more beds for the protracted hospital stay that is often associated with these problems. Centres dealing with complex abdominal surgery, organ transplanta- tion, and hepatic failure will require access to high dependency and intensive treatment beds.

\subsection{OUTPATIENT CLINIC}

Outpatient services form a substantial part of a gastroenterologist's workload and it seems probable that there will be a trend towards the provision of more, but smaller, clinics to permit closer adherence to appointment times and reduce waiting periods in the clinics. New consultant appointments will be required to reduce waiting time in the community. Waiting time to diagnosis will often include a second wait for outpatient endoscopy or radiology. Attempts should be made to minimise this. Close proximity of the outpatient clinic to endoscopy will promote 'same day' investigation by flexible sigmoidoscopy and gastroscopy, which should reduce the time to diagnosis. Provision of such an outpatient service is a desirable goal for medical and surgical gastroenterology.

\subsection{DIAGNOSTIC SERVICES}

\subsubsection{Endoscopy}

The requirements of an endoscopy unit have been described in detail ${ }^{5}$ and should be provided in every district general hospital. The unit should have adequate reception, waiting, and interview/consultation facilities for patients and their relatives. Offices, change, and rest areas are needed for administrative, nursing, and medical staff. Facilities must include an area adjacent to toilets for oral or enema bowel preparation for colonoscopy. The unit should be conveniently situated and adjacent to day care facilities, unless these are fully provided within the endoscopy unit. Because many inpatients are examined, the unit should be within easy access of the wards.

Two endoscopy rooms are required to provide 18 sessions of routine endoscopy. A third procedure room is desirable for flexible sigmoidoscopy and other non-endoscopic gastrointestinal investigations. A central cleaning/ disinfection area must be provided adjacent to the endoscopy room and must be designed to comply with current Control of Substances Hazardous to Health (COSHH) regulations of the Health and Safety Executive.

Endoscopic examinations such as ERCP, oesophageal dilatation and intubation, and certain small bowel intubation investigations require $x$ ray facilities and radiologist support. In an average district general hospital these procedures may require up to two sessions of radiology/week. If ERCP services are provided, two further sessions may be needed. If the department of radiology cannot provide space for these sessions in a convenient site, then provision of a purpose built ERCP room within the endoscopy unit should be considered to permit these facilities to be available at short notice and used flexibly by physicians and surgeons. Alternatively, the endoscopy unit might be equipped with a high quality C-arm image intensifier or screening unit 


\subsubsection{Gastrointestinal measurement laboratory}

A variety of non-endoscopic investigative procedures are now routine in the diagnosis of gastrointestinal disorders (Appendix VI). Ideally such an investigation unit would be located adjacent to the endoscopy unit to avoid duplication of reception and administrative facilities. Every district general hospital would not be required to provide a comprehensive investigative service, local arrangements being made between adjacent districts.

\subsubsection{Radiology}

Although the number of barium meal and barium enema examinations in many districts has declined in recent years radiological examination of the gastrointestinal tract is a vital component of diagnostic services in gastroenterology and hepatology. Every district general hospital requires access to standard barium examinations including barium follow through examination and enteroclysis. Good quality ultrasound and computerised tomography is vital to the investigation of many gastrointestinal and hepatic disorders and should be available in every district general hospital and radiologists here should have expertise in performing biopsies under ultrasound or computed tomography guidance. Not all district general hospitals will provide interventional radiological investigation of the biliary tract although where this is provided appropriate time in screening rooms will be necessary. Intestinal angiography will not be required in every district general hospital but provision for this should be available on a subregional basis. The development of interventional techniques such as therapeutic embolisation and transjugular intrahepatic portosystemic stent shunting (TIPSS) will be limited to specialist units.

\section{Staffing}

\subsection{MEDICAL}

\section{Consultant}

The total number of consultant physicians with a specialist interest in gastroenterology has progressively increased during the past 10 years with an average rate of $3 \%$ (compound)/annum (Appendix I). It is now considered that there should be one gastroenterologist/100 000 population but this target has only been achieved in one region in England and Wales (Appendix II). Many regions have only one/150 000 or more and in some there is only one for over 200000 of population. Thus, there is still a need for new appointments of consultant physicians with a special interest in gastroenterology. A typical consultant contract for physicians with an interest in gastroenterology and hepatology would contain a commitment to:

Patient care - ward rounds and outpatients (4 sessions); endoscopy or other special investigations ( 3 sessions); administration (1 session); audit and teaching (1 session); study and research ( 1 session).

More than $80 \%$ of general physician/ gastroenterologists have a substantial commit- ment to the acute general medical service, on average 1:4.5 days. They provide an emergency endoscopy service for gastrointestinal bleeding, which has become an even greater commitment with the advent of therapeutic techniques for its control and often organise the local nutrition service. Many district general gastroenterologists cannot develop their endoscopic skills as new techniques develop because this is precluded by non-gastrointestinal clinical commitments.

Funior medical staff - (a) senior registrar to provide replacement of consultants; (b) registrars; (c) senior house officers and house officers.

\section{Permanent non-consultant staff}

Associate specialists, hospital practitioners or clinical assistants can make an important contribution to patient care. It is anticipated that such appointments will increase in future years, particularly to reduce waiting times for routine diagnostic and therapeutic endoscopy and to facilitate the wider development of rapid access clinics with same day diagnosis. It is not anticipated that enrolment of staff grades will reduce the need for a continued expansion of consultant posts in gastroenterology.

\subsection{NURSING}

There is no formal training programme for nursing in gastroenterology. Some specialised units, however, have developed local training programmes to establish special nursing expertise. This is highly desirable particularly in well integrated medical/surgical departments of gastroenterology.

\subsection{NURSE SPECIALISTS}

\section{Endoscopy}

Nurses employed in endoscopy units should receive relevant specialised training and the nurse in charge of an endoscopy unit requires training to a higher level so as to ensure high standards and efficiency in the use, maintenance, and disinfection of the complicated equipment and accessories used. There is an English National Board short course in nursing for gastrointestinal endoscopy and related procedures. Nursing staff have an important part to play in the management of the endoscopy unit, including budgetary control, arranging instrument maintenance contracts and repairs, ordering supplies, supervision of secretarial staff, answering queries, overseeing correct data entry, labelling and transmission of specimens and reports, and ensuring that appointments and rebookings are correctly made.

A two roomed endoscopy unit cannot function at peak periods without six trained nurses. Two nurses are needed in each endoscopy room. Another qualified nurse should be responsible for patient preparation and explanation of the procedure and another should supervise recovery unless this is supervised by a nurse in a neighbouring day care unit. Six staff must be employed to provide five on site, permitting $20 \%$ sick, study or annual leave. Time must be allocated 
to the sister in charge for administration and management. A district general hospital providing 10 sessions of routine endoscopy, mainly using one room at a time, but using a second room for emergencies or flexible sigmoidoscopies needs a sister ( $G$ grade), a senior staff nurse ( $F$ grade), and four nurses (E-D grades) to ensure four nurses on duty most of the time and five at times of greatest activity. For larger units, and those providing ERCP or a bronchoscopy commitment, extra endoscopy trained nurses will be needed.

Non-endoscopic gastrointestinal investigations in many units nurses take an active role in the running of a clinical measurement laboratory performing a variety of investigations from breath tests to manometry. No formal training programme exists for these specialist nurses and their relation to the clinical measurement technicians needs to be established.

\section{Nutrition}

An active gastroenterology unit in a district general hospital should have a nurse specialist in nutrition to assist in the assessment of patients' nutritional state and to play an active part in the administration of enteral and parenteral nutritional support in hospital. In addition, the nurse specialist should participate in discharge planning for patients returning to the community with continuing nutritional support and to liaise directly with community nurses for the provision of continuing care.

Stoma care

A district general hospital requires two nurse specialists in stoma care, to provide support before and after surgical care both in hospital and the community.

\section{Gastroenterology specialist nurse practitioner}

This new position, currently undergoing expansion in gastroenterology, will provide similar services to those of diabetic nurse practitioners. Nurse practitioners in gastroenterology will assist in outpatient clinics and wards, counselling patients about their disease and its management. They will liaise with both hospital staff and healthcare workers in the community. This development in gastroenterology services is in line with the document On the Health of the Nation and thought to be an advance in the care offered to patients.

\subsection{ENDOSCOPY DEPARTMENT ASSISTANTS}

In some endoscopy units operating department assistants (ODAs) have been trained to care for endoscopes and their sterilisation. A wider use of an endoscopy department assistant is currently being considered as this would relieve endoscopy nurses of some of their non-patient orientated duties. It is recommended that there should be an expansion of these posts within endoscopy units.

\subsection{CLINICAL MEASUREMENT TECHNICIANS}

Many of the non-endoscopic investigations in gastroenterology are performed by clinical scientists or medical technical officers (currently about 50 in the UK), research nurses (about 25 in the UK) or research fellows. Physiological measurement in gastroenterology is not recognised as a defined clinical need but it is recommended that posts of clinical measurement technicians should be established to perform the variety of physiological and other tests that have become established as an important part of clinical gastroenterology. An appropriate in service training programme has been established, which has been endorsed by the Department of Health.

\subsection{SECRETARIAL/ADMINISTRATION}

Adequate provision must be made for secretarial and administrative support for a gastroenterology service. The secretarial and administrative load in an endoscopy unit is often taken on by the sister in charge. With the increasing demands on the nursing aspects of endoscopy this is no longer acceptable. A standard district general hospital endoscopy unit requires a full time secretary to coordinate appointments, to maintain computer based records to access data for audit, deal with telephone enquiries, and perform reception functions. Large, busy units may require additional clerical support.

\section{Training in gastroenterology}

The training programme for senior registrars in gastroenterology invariably includes a general internal medicine component, which is provided for both in the teaching hospital and district general hospital. Specialist training in gastroenterology will include broad experience in inpatient and outpatient management of gastrointestinal and liver disorders and a broad training in all diagnostic and therapeutic endoscopic techniques including ERCP. The joint planning advisory committee currently accepts that after general professional training candidates would acquire specialist status after six years, of which 2-3 years will be at registrar level, and 3-4 years at senior registrar level. A period of research lasting 2-3 years leading to a postgraduate qualification is highly desirable and is essential for those aiming to work in teaching hospitals and major district general hospitals.

The Royal College of Physicians (London) Committee for gastroenterology supported by the clinical services committee of the BSG are pressing for a shorter revised schedule of training. In essence, after registration, this would provide four years in general medicine and four years in specialist medicine. Selection for all specialities would take place during the final phase of the first four years and the training in specialist medicine would include 12-18 months in some form of research (which might be bioscientific, clinical, epidemiological, or related to methods providing health care). There would be alternative pathways for those seeking an academic or research career.

A working group of specialist medical training under the chairmanship of the Chief Medical Officer published a report in April 1993, Hospital doctors: training for the future. This group 
proposes that the duration of specialist training should be reduced to seven years or less, that there be a unified training grade (career registrar and senior registrar combined) and that a certificate of specialist training (CCST) should be awarded by the General Medical Council on the advice from the appropriate college that a doctor has completed a training programme.

The specific implications of this report for gastroenterology and the future training needs of the speciality will be considered by the newly formed training committee of the BSG.

During the period of clinical training, 20-25\% of a senior registrar's time should be available to continue research and postgraduate education. There should be some flexibility in the training programme depending on whether the candidate wishes to be a general physician with an interest in gastroenterology in a district general hospital, a consultant physician with an interest in gastroenterology in a teaching hospital or an academic gastroenterologist.

\section{Audit}

Regular and accurate audit is essential for maintaining and improving quality of clinical care. Experience with audit of clinical practice within single disciplines has shown wide variations in outcome. The multidisciplinary nature of gastroenterology makes accurate audit a necessity of everyday clinical practice to assess and improve the outcome of jointly managed clinical problems.

There are many areas where quality control in the form of regular audit can be usefully applied. The management of acute upper gastrointestinal haemorrhage where the application of endoscopic therapeutic procedures have reduced operation rates and death need to be assessed within district general hospitals where the gold standard results from specialised centres should be compared with local practice and steps taken to modify treatment protocols where necessary. Guidelines for good practice in audit of the management of upper gastrointestinal haemorrhage have already been established by a joint working part of the BSG, the research unit of the Royal College of Physicians of London, and the audit unit of the Royal College of Surgeons of England. ${ }^{9}$ Management of inflammatory bowel disease, both from the point of view of shared care with primary care practitioners and the joint management with surgeons and physicians to avoid the life threatening complications of the disease should be audited to assess both outcome in terms of mortality and quality of life for the patient.

Intraregional and regional to supraregional audit can identify guidelines for the best clinical practice for management of conditions requiring specialised treatment.

Regular, multidisciplinary audit meetings, including physicians, surgeons, pathologists, and radiologists should be an essential part of all units providing gastroenterology services. Gastroenterology, nationally, already has a sound basis for comparative audit in the multidisciplinary membership of the British Society of Gastroenterology and in the regional gastro- intestinal societies where audit should be a regular agenda item.

\section{Research}

Time and facilities for clinical and basic research should be available for consultants and trainees. Many gastroenterologists will wish to maintain their interests in clinical research, which can be performed within the hospital setting. Some may wish to have laboratory facilities, within gastroenterology or liver units or collaborate with scientists in basic science departments of the university. These activities should be encouraged and fostered.

The British Society of Gastroenterology research advisory committee has developed 'A strategy for research in gastroenterology' and has highlighted two important areas in which research in gastroenterology might be developed further. The committee identified a need to take a more active and direct part in developing research in the basic sciences relevant to gastroenterology and also in health services research and development.

Enhancing relations between basic science and clinical departments both in universities and other institutions - it is recommended that heads of departments should take a lead in developing joint research projects for which joint funding should be sought. In addition, research training fellowships for well motivated young clinicians intent on an academic career should be established and placed largely within basic science departments closely associated with clinical gastroenterology units. For non-clinical researchers appointed to clinical units it is suggested that appointments should be held jointly with bioscience departments and that there should be practical and tangible evidence of the joint nature of these posts. A small number of senior clinical research fellowships should be awarded to leading researchers who are clearly capable of developing strong independent research lines. Within these posts occupants should be able to pursue their research without the pressure of heavy clinical and teaching commitments.

Enhancing health services research and development - The British Society of Gastroenterology has set up a working party to examine ways in which specific research proposals can be formulated in areas of health service needs in gastroenterology. The working party will also make recommendations about the need for training programmes especially designed for researchers in these fields. Future activities of the Society will include a teaching symposium on health services research to discuss the scope of this type of research and how it can be encouraged.

This working party report was accepted by the clinical services committee of the British Society of Gastroenterology and approved by the council of the British Society of Gastroenterology in June 1993 .
by the

Members of the gastroenterology services working party M J G Farthing (chairman), secretary, British Society of Gastroenterology; R Williams, chairman, clinical services committee, BSG; C H J Swan, chairman, endoscopy section BSG A Burroughs, chairman, liver section, BSG; R C Heading,
chairman, oesophageal section, BSG; J A Dodge, chairman, 
paediatric section, BSG; $\mathbf{R}$ I Russell, chairman, small bowel nutrition section, BSG; CW Venables, chairman, surgical section, BSG; R Dick, chairman, radiology section, BSG; R Burnham, manpower, clinical services committee, BSG; R Leicester, audit, clinical services committee, BSG; G Neale, representative of the Royal College of Physicians committee on gastroenterology. Coopted members - E T Swarbrick, Wolverhampton; P D Fairclough, London; R Jones, chairman; Primary Care Society for Gastroenterology; N P Melia, Department of Health.

1 The British Society of Gastroenterology. Provision of gastroenterological service in the district general hospital. London, 1979.

2 Burnham WR, Lennard-Jones JE, Sladen GE. Staffing of a combined general medical service and gastroenterology unit in a district general hospital. Gut 1989; 30: 546-50.

3 Smith PM, Williams R. A comparison of workloads of physiciangastroenterologists and other consultant physicians. $\mathcal{F} R$ Coll Physicians Lond 1992; 26: 167-8.

\section{Appendix I}

NUMBERS AND DISTRIBUTION OF CONSULTANT PHYSICIANS WITH A SPECIAL INTEREST IN GASTROENTEROLOGY (DATA FOR 1980-1991)

\begin{tabular}{lrrrrrr}
\hline Region & 1980 & 1982 & 1986 & 1988 & 1990 & Dec 1991 \\
\hline East Anglia & 7 & 8 & 9 & 11 & 13 & 13 \\
Mersey & 10 & 12 & 12 & 12 & 12 & 16 \\
Northern & 13 & 17 & 19 & 19 & 20 & 19 \\
North West & 20 & 23 & 25 & 27 & 26 & 30 \\
Oxford & 7 & 7 & 9 & 11 & 11 & 11 \\
South West & 11 & 12 & 14 & 14 & 15 & 15 \\
Thames NE & 27 & 33 & 34 & 35 & 40 & 45 \\
Thames NW & 28 & 29 & 27 & 28 & 28 & 27 \\
Thames SE & 22 & 22 & 23 & 26 & 30 & 29 \\
Thames SW & 16 & 16 & 15 & 16 & 17 & 18 \\
Trent & 17 & 18 & 22 & 26 & 24 & 26 \\
Wessex & 13 & 13 & 13 & 15 & 16 & 16 \\
West Midlands & 27 & 27 & 28 & 31 & 31 & 36 \\
Yorkshire & 17 & 22 & 21 & 22 & 21 & 22 \\
Wales & 10 & 11 & 14 & 14 & 15 & 17 \\
Total & 245 & 270 & 286 & 307 & 319 & 340 \\
\hline
\end{tabular}

\section{Appendix II}

REGIONAL DISTRIBUTION OF PHYSICIAN/GASTROENTEROLOGISTS AND RELATION TO POPULATION SIZE (DECEMBER 1991)

\begin{tabular}{lcccll}
\hline & Population & $\begin{array}{l}\text { No of } \\
\text { consultants } \\
\text { Region }\end{array}$ & $\begin{array}{l}\text { No of sessions } \\
\text { (specialist } \\
\text { Gastroenterology) }\end{array}$ & $\begin{array}{l}\text { Population/ } \\
\text { consultant } \\
10^{3}\end{array}$ & $\begin{array}{l}\text { Population/WTE } \\
\text { (7 sessions) } \\
10^{3}\end{array}$ \\
\hline Northern & 3077 & 19 & 96 & 162 & 237 \\
Yorkshire & 3605 & 22 & 107 & 165 & 240 \\
Trent & 4646 & 26 & 145 & 178 & 221 \\
East Anglia & 2014 & 13 & 65 & 154 & 223 \\
Thames NW & 3488 & 27 & 160 & 129 & 152 \\
Thames NE & 3772 & 45 & 249 & 84 & 106 \\
Thames SE & 3635 & 29 & 141 & 126 & 182 \\
Thames SW & 2960 & 18 & 83 & 164 & 247 \\
Wessex & 2905 & 16 & 79 & 182 & 265 \\
Oxford & 2502 & 11 & 67 & 227 & 260 \\
South West & 3206 & 15 & 65 & 214 & 357 \\
West Midlands & 5198 & 36 & 203 & 144 & 179 \\
Mersey & 2409 & 16 & 85 & 151 & 201 \\
North West & 3991 & 30 & 144 & 133 & 194 \\
Wales & 2836 & 17 & 85 estimated & 167 & 235 estimated \\
England and Wales & 50244 & 340 & 1774 & 148 & 198 \\
\hline
\end{tabular}

\section{Appendix III}

\section{DEATHS FROM GASTROINTESTINAL AND LIVER DISORDERS}

\begin{tabular}{llllll}
\hline & 1986 & 1987 & 1988 & 1989 & 1990 \\
\hline GI and liver disorders & 60928 & 61115 & 61784 & 63014 & 58598 \\
\% total deaths & $10 \cdot 5$ & $10 \cdot 8$ & $10 \cdot 8$ & $11 \cdot 1$ & $11 \cdot 1$ \\
GI cancer & 40353 & 40698 & 40986 & 41.135 & 38379 \\
\% total cancer deaths & $29 \cdot 1$ & $29 \cdot 0$ & $28 \cdot 8$ & $28 \cdot 7$ & $28 \cdot 6$ \\
GI infection & 173 & 160 & 162 & $7 \cdot 185$ & \multicolumn{1}{c}{182} \\
\% total infection deaths & $7 \cdot 1$ & $6 \cdot 8$ & $6 \cdot 6$ & $7 \cdot 3$ & $8 \cdot 1$ \\
GI cancer & 4517 & 4762 & 4868 & 5008 & 4890 \\
oesophagus & 9698 & 9496 & 9406 & 9062 & 8085 \\
stomach & 10996 & 11351 & 11464 & 11626 & 10788 \\
colon & 5803 & 5666 & 5751 & 5756 & 5368 \\
anorectum & 6025 & 6045 & 5987 & 6116 & 5762 \\
pancreas & 4522 & 4297 & 4268 & 4399 & 4119 \\
Peptic ulcer & 2007 & 1899 & 1988 & 1989 & 1901 \\
Intestinal obstruction & 2501 & 2672 & 2765 & 3016 & 2823 \\
Liver disease & & & & &
\end{tabular}

OPCS mortality statistics, England and Wales. GI=gastrointestinal. hospital. London: 1992.
4 Holman RAE, Davis M, Gough KR, Gartell P, Britton DC, Smith RB. Value of a centralised approach in the management of haematemesis and melaena:

5 The British Society of Gastroenterology. Provision of gastrointestinal endoscopy and related services for a district general

6 King's Fund Centre. A positive approach to nutrition as treatment. London: 1992.

7 Burnham WR. The role of nutritional support teams. In: Payne-James J, Grimble G, Silk D, eds. Artificial nutrition support in clinical practice. London: Edward Arnold, 1994 (in press). services for paediatric gastroenterology and nutrition. Report terology and Nutrition.

Working Group. Guideliness for good practice in and rhage. $\mathcal{F} R$ Coll Physicians Lond 1992; 26: 281-9. 


\section{Appendix IV}

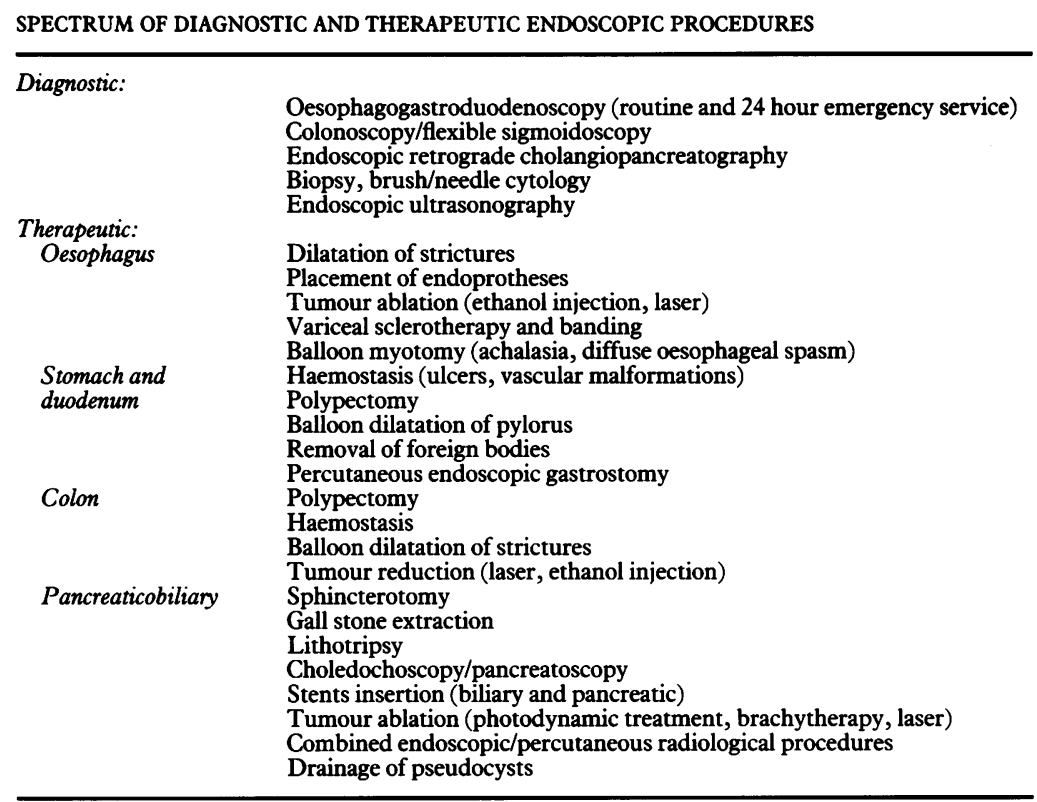

\section{Appendix V}

AVAILABILITY OF ENDOSCOPIC PROCEDURES IN THE UK $\star$

\begin{tabular}{|c|c|c|}
\hline & $\begin{array}{l}\text { No of hospitals } \\
(\%)\end{array}$ & $\begin{array}{l}\text { Open access to } \\
\text { general practitioners }\end{array}$ \\
\hline \multicolumn{3}{|l|}{ Diagnostic upper GI } \\
\hline $\begin{array}{l}\text { endoscopy } \\
\text { Variceal injection }\end{array}$ & $\begin{array}{l}201(99) \\
176(86)\end{array}$ & $121(59)$ \\
\hline Haemostasis & $158(78)$ & - \\
\hline Balloon myotomy & $139(68)$ & - \\
\hline Diagnostic colonoscopy & $198(97)$ & $18(9)$ \\
\hline Polypectomy & $198(97)$ & - \\
\hline Haemostasis & $124(61)$ & \\
\hline Balloon dilatation & $86(42)$ & \\
\hline Flexible sigmoidoscopy & $176(98)$ & $28(13)$ \\
\hline Diagnostic ERCP & $156(79)$ & - \\
\hline Sphincterotomy & $150(74)$ & - \\
\hline Stone removal & $139(68)$ & - \\
\hline Stent placement & $120(59)$ & - \\
\hline
\end{tabular}

* Data for 204 hospitals in England, Wales, Scotland and Northern Ireland. $\mathrm{GI}=$ gastrointestinal; ERCP=endoscopic retrograde cholangiopancreatography.

\section{Appendix VI}

NON-ENDOSCOPIC GASTROINTESTINAL INVESTIGATIONS

\begin{tabular}{|c|c|}
\hline $\begin{array}{l}\text { Gastrointestinal secretion } \\
\text { and absorption: }\end{array}$ & $\begin{array}{l}\text { Gastric secretion } \\
\text { Pancreatic secretion (Lundh, CCK/PZ) } \\
\text { Secretin test (gastrinoma) } \\
\text { Intestinal perfusion tests } \\
\text { Vitamin B-12 absorption }\end{array}$ \\
\hline Gastrointestinal motility: & $\begin{array}{l}\text { Gastric emptying } \\
\text { Intestinal transit }\end{array}$ \\
\hline & $\begin{array}{l}\text { Oesophageal manometry } \\
\text { Small intestinal manometry }\end{array}$ \\
\hline Gastro-oesophageal reflux: & $\begin{array}{l}24 \text { hour ambulatory oesophageal pH monitoring } \\
\text { Radionuclide scanning }\end{array}$ \\
\hline Visceral sensation: & $\begin{array}{l}\text { Rectal sensation } \\
\text { Bernstein test }\end{array}$ \\
\hline $\begin{array}{l}\text { Tissue biopsy: } \\
\text { Bacterial overgrowth: }\end{array}$ & $\begin{array}{l}\text { Intestine, liver, pancreas, etc } \\
\text { Hydrogen breath tests } \\
\text { Duodenal intubation }\end{array}$ \\
\hline H pylori testing: & $\begin{array}{l}{ }^{13} \mathrm{C} \text { or }{ }^{14} \mathrm{C} \text { urea breath tests } \\
\text { Serology }\end{array}$ \\
\hline
\end{tabular}

\section{Appendix VIII}

CONDITIONS THAT COULD BE REFERRED TO REGIONAL PAEDIATRIC CENTRES

AVAILABILITY OF NON-ENDOSCOPIC GASTROINTESTINAI INVESTIGATIONS IN THE UK ${ }^{\star}$

\begin{tabular}{lc}
\hline & No of hospitals $(\%)$ \\
\hline Oesophageal manometry & $33(16)$ \\
Ambulatory oesophageal pH & $86(42)$ \\
Gastric emptying & $53(26)$ \\
$\quad$ radionuclide & $27(13)$ \\
ultrasound & $22(11)$ \\
Anorectal manometry & $7(1 \cdot 4)$ \\
Electromyography & $58(28)$ \\
Hydrogen breath tests & $70(34)$ \\
glucose & $81(40)$ \\
lactulose & $53(26)$ \\
Gastric secretion & \\
Pancreatic secretion & $65(32)$ \\
H pylori tests & $186(91)$ \\
Serology & $142(70)$ \\
Antral biopsy, culture/history & $42(21)$ \\
Urease test & i'C or ${ }^{13} \mathrm{C}$ urea breath test
\end{tabular}

^ Data for 204 hospitals in England, Wales, Scotland and Northern Ireland.
Inflammatory bowel disease

Protracted diarrhoea/vomiting

Children requiring highly specialised diets

Children requiring parenteral and enteral nutrition

Children requiring specialised investigations including endoscopy and jejunal biopsy

Investigation of gastrointestinal bleeding

Severe refractory constipation

Rare conditions such as chronic intestinal pseudoobstruction and congenital defects in intestinal transport

9 All forms of chronic liver disease

Patients with fulminant liver failure or subacute liver failure should be referred directly to a supraregiona centre offering intensive care and liver transplantation 